\title{
High prevalence of hip lesions secondary to arthroscopic over- or undercorrection of femoroacetabular impingement in patients with postoperative pain
}

\author{
Malin K. Meier ${ }^{1} \cdot$ Till D. Lerch $^{2} \cdot$ Simon D. Steppacher $^{1} \cdot$ Klaus A. Siebenrock $^{1} \cdot$ Moritz Tannast $^{3} \cdot$ Peter Vavron $^{4}$. \\ Ehrenfried Schmaranzer ${ }^{5} \cdot$ Florian Schmaranzer $^{2,5}$ (i)
}

Received: 12 August 2021 / Revised: 28 September 2021 / Accepted: 27 October 2021 / Published online: 29 November 2021

(c) The Author(s) 2021

\begin{abstract}
Objectives To compare the prevalence of pre- and postoperative osseous deformities and intra-articular lesions in patients with persistent pain following arthroscopic femoroacetabular impingement (FAI) correction and to identify imaging findings associated with progressive cartilage damage.

Methods Retrospective study evaluating patients with hip pain following arthroscopic FAI correction between 2010 and 2018. Pre- and postoperative imaging studies were analyzed independently by two blinded readers for osseous deformities (cam-deformity, hip dysplasia, acetabular overcoverage, femoral torsion) and intra-articular lesions (chondro-labral damage, capsular lesions). Prevalence of osseous deformities and intra-articular lesions was compared with paired $t$-tests/McNemar tests for continuous/dichotomous data. Association between imaging findings and progressive cartilage damage was assessed with logistic regression.

Results Forty-six patients (mean age 29 \pm 10 years; 30 female) were included. Postoperatively, 74\% (34/46) of patients had any osseous deformity including 48\% (22/46) acetabular and femoral deformities. Ninety-six percent (44/46) had an intra-articular lesion ranging from $20 \%$ (9/46) for femoral to 65\% (30/46) for acetabular cartilage lesions. Prevalence of hip dysplasia increased ( 2 to $20 \%, p=0.01$ ) from pre- to postoperatively while prevalence of cam-deformity decreased ( 83 to $28 \%, p<0.001)$.

Progressive cartilage damage was detected in 37\% (17/46) of patients and was associated with extensive preoperative cartilage damage $>2$ h, i.e., $>60^{\circ}$ (OR 7.72; $p=0.02$ ) and an incremental increase in postoperative alpha angles (OR 1.18; $\left.p=0.04\right)$. Conclusion Prevalence of osseous deformities secondary to over- or undercorrection was high. Extensive preoperative cartilage damage and higher postoperative alpha angles increase the risk for progressive degeneration.

Key Points • The majority of patients presented with osseous deformities of the acetabulum or femur (74\%) and with intraarticular lesions (96\%) on postoperative imaging.

- Prevalence of hip dysplasia increased (2 to 20\%, $p=0.01$ ) from pre-to postoperatively while prevalence of a cam deformity decreased ( 83 to $28 \%, p<0.001$ ).

- Progressive cartilage damage was present in $37 \%$ of patients and was associated with extensive preoperative cartilage damage $>2 h(O R 7.72 ; p=0.02)$ and with an incremental increase in postoperative alpha angles $($ OR 1.18; $p=0.04)$.
\end{abstract}

Keywords MR arthrography $\cdot$ Hip arthroscopy $\cdot$ Cartilage $\cdot$ Hip $\cdot$ Femoroacetabular impingement

Florian Schmaranzer

florian.schmaranzer@insel.ch

1 Department of Orthopedic Surgery and Traumotology, Inselspital Bern University Hospital, University of Bern, Freiburgstrasse, 3010 Bern, Switzerland

2 Department of Diagnostic-, Interventional- and Pediatric Radiology, Inselspital Bern University Hospital, University of Bern, Freiburgstrasse, 3010 Bern, Switzerland
3 Department of Orthopaedic Surgery, Fribourg Cantonal Hospital, University of Fribourg, Chemin des Pensionnats 2-6, 1752 Villars-sur-Glâne, Switzerland

4 Department of Orthopaedic Surgery, District Hospital St. Johann in Tirol, Bahnhofstrasse 14, 6380 St. Johann in Tirol, Austria

5 Department of Radiology, District Hospital St. Johann in Tirol, Bahnhofstrasse 14, 6380 St. Johann in Tirol, Austria 


\section{Abbreviations}

AP Anteroposterior

CI Confidence interval

FAI Femoroacetabular impingement

LCE Lateral center edge angle

OR Odds ratio

\section{Introduction}

Recent years have led to an exponential increase in the number of hip arthroscopies performed for treatment of osseous deformities and intra-articular lesions secondary to femoroacetabular impingement [1]. This development has been fostered by innovations in surgical techniques, improved preoperative imaging, and the widely reported short- to long-term benefit of FAI surgery [2-4]. Despite the favorbale surgical outcome of FAI correction up to $18 \%$ of patients present with postoperative pain and up to $10 \%$ reportedly undergo revision surgery within 2 years [5, 6]. These patients are exposed to a substantially higher risk for worse surgical outcome following revision surgery than patients who do not require further surgical treatment [7]. Further cross-sectional imaging is commonly recommended to diagnose deformities resulting from surgical over- or undercorrection such as residual cam deformities and intra-articular lesions $[8,9]$. However, imaging assessment is challenging in the setting of postoperative hip pain due to the difficulties in identifying the relevant osseous deformities and differentiating normal postoperative findings from relevant pathology $[8,10,11]$.

Currently, few studies have assessed the prevalence of osseous deformities following arthroscopic FAI surgery with cross-sectional imaging [12]. MR arthrography has been used in the setting of postoperative hip pain to detect recurrent labrum lesions, cartilage damage, and capsular lesions [10, 13-15]. However, to date, the prevalence of osseous deformities and intra-articular lesions including their potential association following failed hip arthroscopy is unclear which would provide useful information for treatment planning.

Thus, the aim of this study was to compare the prevalence of osseous deformities and intra-articular lesions in patients with persistent pain following arthroscopic FAI correction between pre- and postoperative imaging and to identify imaging findings associated with progressive cartilage damage and postoperative labrum lesions.

\section{Material and methods}

\section{Patients}

Following IRB approval and a waiver for written informed consent, a retrospective study was performed at a primary orthopedic hospital. The imaging database was searched for consecutive patients originating from Western Austria who had undergone arthroscopic FAI correction and preoperative radiographic and traction MR arthrography according to the institutional routine protocol between January 2010 and 2018. Inclusion criterion was availability of postoperative imaging including radiographs and traction MR arthrography for refractory hip pain. Patients were excluded if no sequences covering the distal femoral condyles for measurement of femoral torsion were available. Diagnosis of hip pain was established by two arthroscopic hip surgeons based on a $>3$-month history of symptoms and a positive impingement test $[2,14]$.

\section{Diagnostic imaging}

AP pelvis and Dunn $45^{\circ}$ views were obtained in supine position pre- and postoperatively [16]. Pre- and postoperative MR arthrography was performed at $1.5 \mathrm{~T}$ (Magnetom Symphony/Aera; Siemens Healthineers) following fluoroscopic injection of 1-2 $\mathrm{ml}$ of iodinated contrast agent (iopamidol, $200 \mathrm{mg} / \mathrm{ml}$; Iopamiro 200; Bracco), 2-5 ml of local anesthetic (ropivacaine hydrochloride; $2 \mathrm{mg} / \mathrm{ml}$; Ropinaest;Gebro Pharma), and 15-20 ml of diluted MR contrast agent (gadopentetate dimeglumine, $2 \mathrm{mmol} / \mathrm{l}$; Magnevist; Bayer Healthcare). As part of the institutional routine protocol, leg traction was applied during MRI using a previously described method and a dedicated traction device (TRACView; Menges Medical) [17, 18]. This includes a supporting plate for stabilization of the contralateral leg, a weight (adjusted to patients constitution: $15 \mathrm{~kg}$ for patients $<60 \mathrm{~kg}, 18 \mathrm{~kg}$ for patients $60-80 \mathrm{~kg}, 23 \mathrm{~kg}$ for patients $>80 \mathrm{~kg}$ ) connected to a cable whinch via a pulley which is connected to an ankle brace. The preoperative imaging protocol included multiplanar (coronal, sagittal, and axial-oblique) gradient echo- or turbo spin echo sequences and 3D sequences for reformation of radial images with a total imaging time of $21-23 \mathrm{~min}$. The postoperative imaging protocol included multiplanar turbo spin echo sequences and 3D sequences for reformation of radial images. In addition, axial sequences of the pelvis and distal femoral condyles were acquired without leg traction for measurement of femoral torsion leading to a total imaging time of 23-25 min. Imaging protocol is given in Supplementary Table 1.

\section{Image analysis}

Analysis of pre- and postoperative imaging was performed independently by two blinded readers (radiologist with 12 years (E.S.) and resident with 7 years (F.S.) of experience in hip imaging). Pre-and postoperative imaging studies were compared directly against each other, blinded to the operative records. Radiographs were assessed for Tönnis grade of 
osteoarthritis, lateral center edge (LCE) angle according to Wiberg et al. [19], acetabular index, and acetabular retroversion signs (cross over, posterior wall, ischial spine signs) $[16,20]$. Diagnosis of osseous deformities was made according to the 2020 Lisbon agreement on FAI imaging [20]: hip dysplasia $=\mathrm{LCE}<25^{\circ}$, mild acetabular overcoverage LCE $34-40^{\circ}$, severe acetabular overcoverage $=\mathrm{LCE}>40^{\circ}$, acetabular retroversion $=$ presence of all 3 retroversion signs. On radial images, maximum alpha angles were measured and angles $>60^{\circ}$ were consistent with a cam deformity [20]. Femoral torsion was measured according to the method described by Murphy et al. [21] and angles $<0^{\circ}$ and $>35^{\circ}$ were used to diagnose femoral retrotorsion and excessively high femoral torsion, respectively [22].

Acetabular and femoral cartilage damage was consistent with delamination, thinning, or defect [23]. Presence of extensive cartilage damage $>2 \mathrm{~h}$ on the clock face (i.e., $>60^{\circ}$ ) was recorded as it has been linked with failure of FAI surgery [20,24]. Pre- and postoperative imaging was compared to assesses progressive cartilage damage which was defined as any new acetabular/femoral cartilage lesion on postoperative imaging and extension of cartilage damage $>2 \mathrm{~h}$ in a patient with cartilage damage $<2 \mathrm{~h}$ on preoperative imaging. Diagnosis of a postoperative labrum lesion was made as described previously [15]: contrast extension to the labrum surface, presence of paralabral cyst or extension of labrum abnormality to a new location on postoperative MRI. Presence or absence of capsular defects and adhesions was assessed [11]. Obliteration of the paralabral sulcus was not recorded as it reportedly is a uniform postoperative finding [11].

\section{Statistical analysis}

Normal distribution of continuous data was confirmed using Kolmogorov Smirnov test.

Prevalence of osseous deformities and intra-articular lesions was compared pre- and postoperatively with paired $t$-tests for continuous and $\mathrm{McNemar}$ tests for dichotomous data, respectively. Post hoc sample size calculation was performed for assessment of interrater reliability using Cohen's kappa (к). Fair agreement consistent with a $\kappa>0.2$ was chosen as minimum level of agreement. Substantial agreement corresponding to a $\kappa$ of 0.7 was defined as expected interrater agreement [25]. Assuming a significance level of 0.05 , a power of 0.80 and an expected proportion ranging from 0.2 to 0.8 to account for the multiple outcome parameters led to a minmum sample size of 40 hips [26]. After confirmation of at least moderate $(\kappa>0.4)$ interrater reliability for all parameters, results of reader 1 were used for logistic regression analysis [25]. Association of progressive cartilage damage and postoperative labrum lesions with demographic factors and imaging findings was evaluated with odds ratios and corresponding 95\% confidence intervals (CIs) using logistic regression analyis. If more than two significant associations were found, a multivariate logistic regression analysis was performed. A type I error rate of 5\% was used to determine statistical significance. Statistical analysis was performed using GraphPad Prism (Version 9.1, GraphPad Software).

\section{Results}

\section{Patient characteristics}

Of the 806 patients with preoperative imaging and subsequent hip arthroscopy at our institution, 72 patients met the inclusion criteria. Twenty-six patients were excluded due to lack of images of the distal femoral condyles. Finally, 46 patients (46 hips, 30 female) with a mean age at surgery of $29 \pm 10$ years (age range, $16-54$ years) and complete preand postoperative imaging were included who underwent different arthroscopic procedures (Table 1). Mean time between surgery and postoperative MRI was $1.4 \pm 1.0$ years (range 0.1-4.9 years). Seventeen percent (8/46) had revision surgery $1.6 \pm 0.8$ years after the index procedure and $7 \%$ (3/46) underwent subsequent total hip replacement within 2 years (Table 1 ).

Table 1 Demographic characteristics

\begin{tabular}{ll}
\hline Characteristic & $\begin{array}{l}\text { Study group } 46 \\
\text { patients (46 hips) }\end{array}$ \\
\hline Age at surgery (year) & $29 \pm 10$ \\
Time between surgery and second MRI (year) & $1.4 \pm 1$ \\
Female sex & $65 \%(30 / 46)$ \\
Surgical procedure & \\
Cam resection & $93 \%(43 / 46)$ \\
Labrum debridement & $11 \%(5 / 46)$ \\
Labrum refixation & $76 \%(35 / 46)$ \\
Labrum reconstruction & $9 \%(4 / 46)$ \\
Acetabular rim trimming & $72 \%(33 / 46)$ \\
Subspine decompression & $2 \%(1 / 46)$ \\
Cartilage repair & $5 \%(2 / 46)$ \\
Subsequent revision surgery & $17 \%(8 / 46)$ \\
Cam resection & $38 \%(3 / 8)$ \\
Labrum debridement & $50 \%(4 / 8)$ \\
Labrum refixation & $50 \%(4 / 8)$ \\
Labrum reconstruction & $13 \%(1 / 8)$ \\
Acetabular rim trimming & $50 \%(4 / 8)$ \\
Adhesioloysis and caspsular closure & $88 \%(7 / 8)$ \\
Derotational femoral osteotomy & $13 \%(1 / 8)$ \\
Subsequent total hip arthroplasty & $7 \%(3 / 46)$ \\
\hline
\end{tabular}




\section{Prevalence of osseous deformities}

Postoperatively, 74\% (reader 1, 34/46) and 65\% (reader 2, $30 / 46$ ) of patients had an osseous deformity of the acetabulum or the femur. An acetabular deformity was found in $48 \%$ (22/46) of patients for reader 1 and $41 \%$ (19/46) of patients for reader 2 (Table 2). A femoral deformity was found in $48 \%(22 / 46)$ of patients for reader 1 and $41 \%(19 / 46)$ of patients for reader 2 (Table 2).

The prevalence of hip dysplasia $\left(\mathrm{LCE}<25^{\circ}\right)$ increased significantly (both readers, $p=0.01$ ) in reader $1 / 2$ from $2 \%$ (1/46)/4\% (2/46) preoperatively to $20 \%(9 / 46) / 22 \%(10 / 46)$ postoperative, corresponding to a difference of $18 \%(95 \%$ CI, 7-29\%) (Figs. 1, 2). This was reflected by a significant (both readers $p<0.001$ ) decrease in mean LCE angle of $3 \pm 4^{\circ}$ for reader $1\left(95 \% \mathrm{CI}, 2-5^{\circ}\right)$ and reader $2(95 \% \mathrm{CI}$, 2-4 $4^{\circ}$ (Table 2).

Readers 1 and 2 found a significant (both readers, $p<0.001$ ) decrease of 55\% (95\% CI, 41-69\%) and 54\%
(95\% CI, 40-68\%) in the prevalence of a cam deformity from $83 \%$ (38/46) and $76 \%$ (35/46) preoperatively to $28 \%$ (13/46) and 22\% (10/46) postoperatively (Fig. 3). Accordingly, a mean decrease in alpha angles of $11 \pm 11^{\circ}(95 \% \mathrm{CI}$, $\left.7-14^{\circ} ; p<0.001\right)$ and $10 \pm 11^{\circ}\left(95 \% \mathrm{CI}, 7-14^{\circ} ; p<0.001\right)$ was observed by readers 1 and 2, respectively (Table 2 ). Mean femoral torsion was $24 \pm 11^{\circ}$ for reader 1 and $26 \pm 12^{\circ}$ for reader 2 . The prevalence of exccessivley high femoral torsion $\left(>35^{\circ}\right)$ was $24 \%(11 / 46)$ for reader 1 and $20 \%(9 / 46)$ for reader 2 (Table 2, Fig. 4). The prevalence of femoral retrotorsion $\left(<0^{\circ}\right)$ was $4 \%(2 / 46)$ for both readers (Table 2$)$.

\section{Prevalence of intra-articular lesions}

Postoperatively, 96\% (reader 1, 44/46) and 98\% (reader 2, $45 / 46$ ) of patients had any intra-articular lesion ranging from $20 \%$ (reader $1,9 / 46$ ) and $17 \%$ (reader $2,8 / 46$ ) for femoral cartilage lesions to $65 \%$ (reader $1,30 / 46$ ) and $80 \%$ (reader $2,37 / 46$ ) for acetabular cartilage lesions. Preoperatively,

Table 2 Comparison of prevalence between preoperative and postoperative osseous deformities and intra-articular lesions

\begin{tabular}{|c|c|c|c|c|c|c|c|c|}
\hline \multirow[t]{2}{*}{ Parameter } & \multicolumn{3}{|l|}{ Reader 1} & \multirow[b]{2}{*}{$p$ value } & \multicolumn{3}{|l|}{ Reader 2} & \multirow[b]{2}{*}{$p$ value } \\
\hline & Preoperative & Postoperative & Difference & & Preoperative & Postoperative & Difference & \\
\hline \multicolumn{9}{|l|}{ Osseous deformities } \\
\hline Tönnis grade $=1$ & $30 \%(14)$ & $41 \%(19)$ & $11 \%(2-20 \%)$ & 0.06 & $21 \%(10)$ & $33 \%(15)$ & $12 \%(3-21 \%)$ & 0.06 \\
\hline $\operatorname{LCE}\left(^{\circ}\right)$ & $34 \pm 6$ & $31 \pm 7$ & $3 \pm 4(2-5)$ & $<0.001$ & $132 \pm 6$ & $29 \pm 7$ & $3 \pm 4(2-4)$ & $<0.001$ \\
\hline Hip dysplasia $\left(\mathrm{LCE}<25^{\circ}\right)$ & $2 \%(1)$ & $20 \%(9)$ & $18 \%(7-29 \%)$ & 0.01 & $4 \%(2)$ & $22 \%(10)$ & $18 \%(7-29 \%)$ & 0.01 \\
\hline $\begin{array}{l}\text { Mild overcoverage (LCE } \\
34-40^{\circ} \text { ) }\end{array}$ & $22 \%(10)$ & $15 \%(7)$ & $7 \%(0-14 \%)$ & 0.51 & $17 \%(8)$ & $13 \%(6)$ & $4 \%(-2-10 \%)$ & 0.69 \\
\hline $\begin{array}{l}\text { Severe overcoverage } \\
\left(\mathrm{LCE}>40^{\circ}\right)\end{array}$ & $20 \%(9)$ & $13 \%(6)$ & $7 \%(0-14 \%)$ & 0.25 & $15 \%(7)$ & $9 \%(4)$ & $6 \%(0-13 \%)$ & 0.25 \\
\hline Acetabular index $\left({ }^{\circ}\right)$ & $4 \pm 5$ & $6 \pm 5$ & $2 \pm 6(0-4)$ & 0.04 & $5 \pm 4$ & $6 \pm 5$ & $1^{\circ} \pm 4^{\circ}(0-2)$ & 0.03 \\
\hline Acetabular retroversion & $13 \%(6)$ & $2 \%(1)$ & $11 \%(2-20 \%)$ & 0.06 & $15 \%(7)$ & $4 \%(2)$ & $11 \%(2-20 \%)$ & 0.06 \\
\hline Crossover sign & $65 \%(30)$ & $52 \%(24)$ & $13 \%(3-23 \%)$ & 0.03 & $63 \%(29)$ & $41 \%(19)$ & $22 \%(10-34 \%)$ & 0.01 \\
\hline Posterior wall sign & $57 \%(26)$ & $52 \%(24)$ & $5 \%(0-11 \%)$ & 0.63 & $41 \%(19)$ & $28 \%(13)$ & $13 \%(3-23 \%)$ & 0.03 \\
\hline Ischial spine sign & $33 \%(15)$ & $33 \%(15)$ & 0 & 1.00 & $28 \%(13)$ & $22 \%(10)$ & $6 \%(0-13 \%)$ & 0.38 \\
\hline Alpha angle $\left(^{\circ}\right)$ & $71 \pm 9$ & $60 \pm 11$ & $11 \pm 11(7-14)$ & $<0.001$ & $170 \pm 11$ & $60 \pm 10$ & $10 \pm 11(7-14)$ & $<0.001$ \\
\hline Cam deformity & $83 \%(38)$ & $28 \%(13)$ & $55 \%(41-69 \%)$ & $<0.001$ & $176 \%(35)$ & $22 \%(10)$ & $54 \%(40-68 \%)$ & $<0.001$ \\
\hline Femoral torsion $\left({ }^{\circ}\right)$ & & $24 \pm 11$ & & & & $26 \pm 12$ & & \\
\hline $\begin{array}{l}\text { Increased femoral torsion } \\
\left(>35^{\circ}\right)\end{array}$ & & $24 \%(11)$ & & & & $20 \%(9)$ & & \\
\hline $\begin{array}{l}\text { Decreased femoral torsion } \\
\left(<0^{\circ}\right)\end{array}$ & & $4 \%(2)$ & & & & $4 \%(2)$ & & \\
\hline \multicolumn{9}{|l|}{ Intra-articular lesions } \\
\hline Labrum lesion & $93 \%(43)$ & $48 \%(22)$ & $45 \%(31-59 \%)$ & $<0.001$ & $198 \%(45)$ & $50 \%(23)$ & $48 \%(34-62 \%)$ & $<0.001$ \\
\hline Acetabular cartilage lesion & $54 \%(25)$ & $65 \%(30)$ & $11 \%(2-20 \%)$ & 0.06 & $70 \%(32)$ & $80 \%(37)$ & $10 \%(1-19 \%)$ & 0.06 \\
\hline Cartilage lesions $>2 \mathrm{~h}$ & $24 \%(11)$ & $37 \%(17)$ & $13 \%(3-23 \%)$ & 0.07 & $20 \%(9)$ & $41 \%(19)$ & $21 \%(9-33 \%)$ & 0.01 \\
\hline Femoral cartilage lesion & $7 \%(3)$ & $20 \%(9)$ & $13 \%(3-23 \%)$ & 0.03 & $9 \%(4)$ & $17 \%(8)$ & $9 \%(1-17 \%)$ & 0.13 \\
\hline Progressive cartilage damage & - & $37 \%(17)$ & - & - & - & $37 \%(17)$ & - & - \\
\hline Capsular defect & - & $48 \%(22)$ & - & - & - & $59 \%(27)$ & - & - \\
\hline Capsular adhesion & - & $39 \%(18)$ & - & - & - & $48 \%(22)$ & - & - \\
\hline
\end{tabular}

LCE lateral center edge angle 
Fig. 1 A 23-year-old man with persisting pain 14 months after arthroscopic cam resection and acetabular rim trimming with labrum refixation. a Preoperative AP pelvis view shows normal lateral coverage (LCE: $27^{\circ}$, a cross-over sign, and a cam deformity. b Preoperative coronal T1-w TSE image (repetition time/echo time, $450 \mathrm{~ms} / 12 \mathrm{~ms}$ ) shows labrum lesion (filled arrow head) and cartilage delamination (arrowhead). c Postoperative AP pelvis view shows a dysplastic actabulum (LCE: $20^{\circ}$ ) following overcorrection. d Postoperative coronal PD-w TSE image (2460 ms/ $13 \mathrm{~ms}$ ) shows large cartilage flap (white arrowhead) and new femoral cartilage defect (black arrowheads) indicating progressive cartilage damage. Note: anterior acetabular wall (red dotted line), posterior acetabular wall (blue dotted line)
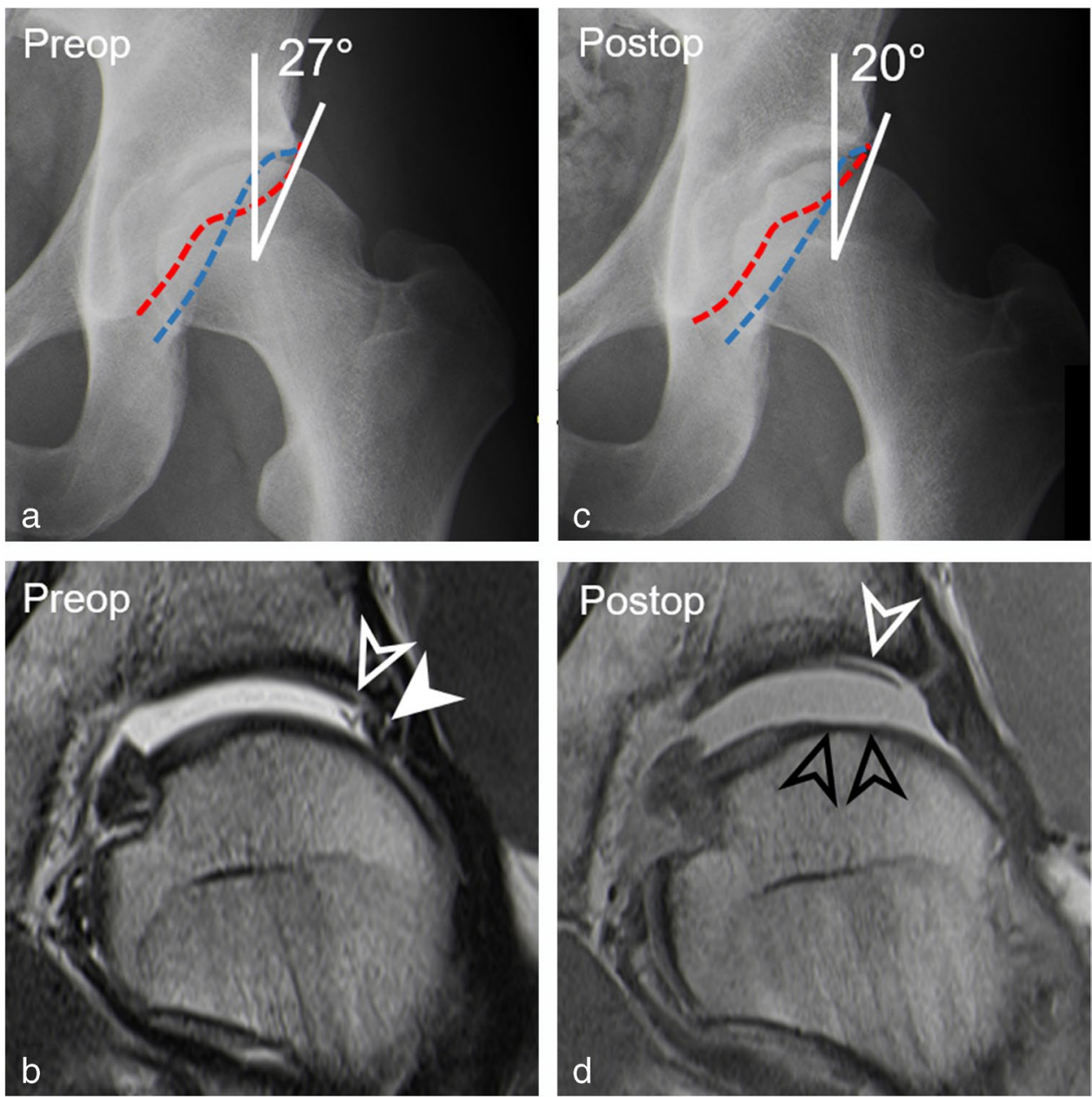

readers 1 and 2 detected a labrum lesion in 93\% (43/46) and $98 \%$ (45/46) of patients. Postoperatively, the prevalence decreased to $48 \%$ (22/46) and 50\% (23/46) corresponding to a significant decrease of $45 \%(95 \% \mathrm{CI}, 31-59 \%, p<0.001)$ and $48 \%$ (95\% CI, 34-62\%; $p<0.001)$ for readers 1 and 2 , respectively (Table 2 ).

Both readers detected progressive cartilage damage in $37 \%$ (17/46) of the patients.

The prevalence of extensive cartilage lesions $>2 \mathrm{~h}$ increased from $24 \%$ (11/46) and 20\% (9/46) preoperatively to $37 \%(17 / 46)$ and $41 \%(19 / 46)$ for reader 1 (difference of $13 \%, 95 \% \mathrm{CI}, 3-23 \% ; p=0.07$ ) and reader 2 (difference of $21 \%, 95 \% \mathrm{CI}, 9-33 \% ; p=0.01)$ on postoperative imaging (Table 2).

A capsular defect was found postoperatively in $48 \%$ (22/46) and 59\% (27/47) of patients for readers 1 and 2 , respectively. Capsular adhesions were present postoperatively in $39 \%$ (18/46) and $48 \%$ (22/46) of patients for reader 1 and reader 2, respectively (Fig. 2).

Femoral neck fractures or femoral head necrosis were not detected.

\section{Interrater reliability}

Interrater reliability for preoperative diagnosis of osseous deformities ranged from $\kappa$ of 0.66 for hip dysplasia to $\kappa$ of 0.91 for acetabular retroversion. Postoperatively, $\kappa$ ranged from 0.60 for presence of cam deformity to 1.00 for diagnosis of femoral retrotrosion. Interrater reliability for detection of preoperative intra-articular lesions ranged from $\kappa$ of 0.51 to 0.85 for diagnosis of acetabular/femoral cartilage lesions. Postoperatively, interrater reliability ranged from 0.52 for diagnosis of acetabular cartilage lesions to 0.82 for diagnosis of capsular adhesions (Table 3 ).

\section{Association between osseous deformities and intraarticular lesions}

Multivariate analysis showed that extensive preoperative cartilage damage $>2 \mathrm{~h}$ (OR [odds ratio] 7.72, 95\% CI 1.52-50.28; $p=0.02$ ) and an incremental increase in postoperative alpha angles (OR $1.18,95 \%$ CI $1.03-1.44 ; p=0.04$ ) was associated with progressive cartilage damage (Table 4, Figs. 3, 5). Postoperative hip dysplasia was the only parameter associated (OR 5.13, 95\% CI 1.07-37.76; $p=0.04$ ) with presence of postoperative labrum lesions (Table 5, Fig. 6). 
Fig. 2 A 29-year-old patient presenting with residual pain 2 years after arthroscopic trimming of the retroverted acetabulum, labrum refixation, and cam resection. a Preoperative AP pelvis view shows normal lateral coverage (LCE: $28^{\circ}$ ) but a retroverted acetabulum. b Preoperative axial-olbique and c coronal PD-w TSE images (repetition time/echo time, $2460 \mathrm{~ms} / 13 \mathrm{~ms}$ ) show anterior labrum tear (filled arrowhead) and intact cartilage. d Postoperative AP pelvis view shows deficient acetabular coverage (LCE: $22^{\circ}$ ) following acetabular overcorrection. e Postoperative axial-oblique and $\mathbf{f}$ coronal PD-w TSE images (repetition time/echo time, $2460 \mathrm{~ms} / 13 \mathrm{~ms}$ ) show capsular adhesions (white arrows) and capsular defect (asterisks) and new femoral cartilage delamination (black arrowhead). Note: ischial spine sign (white dotted line)
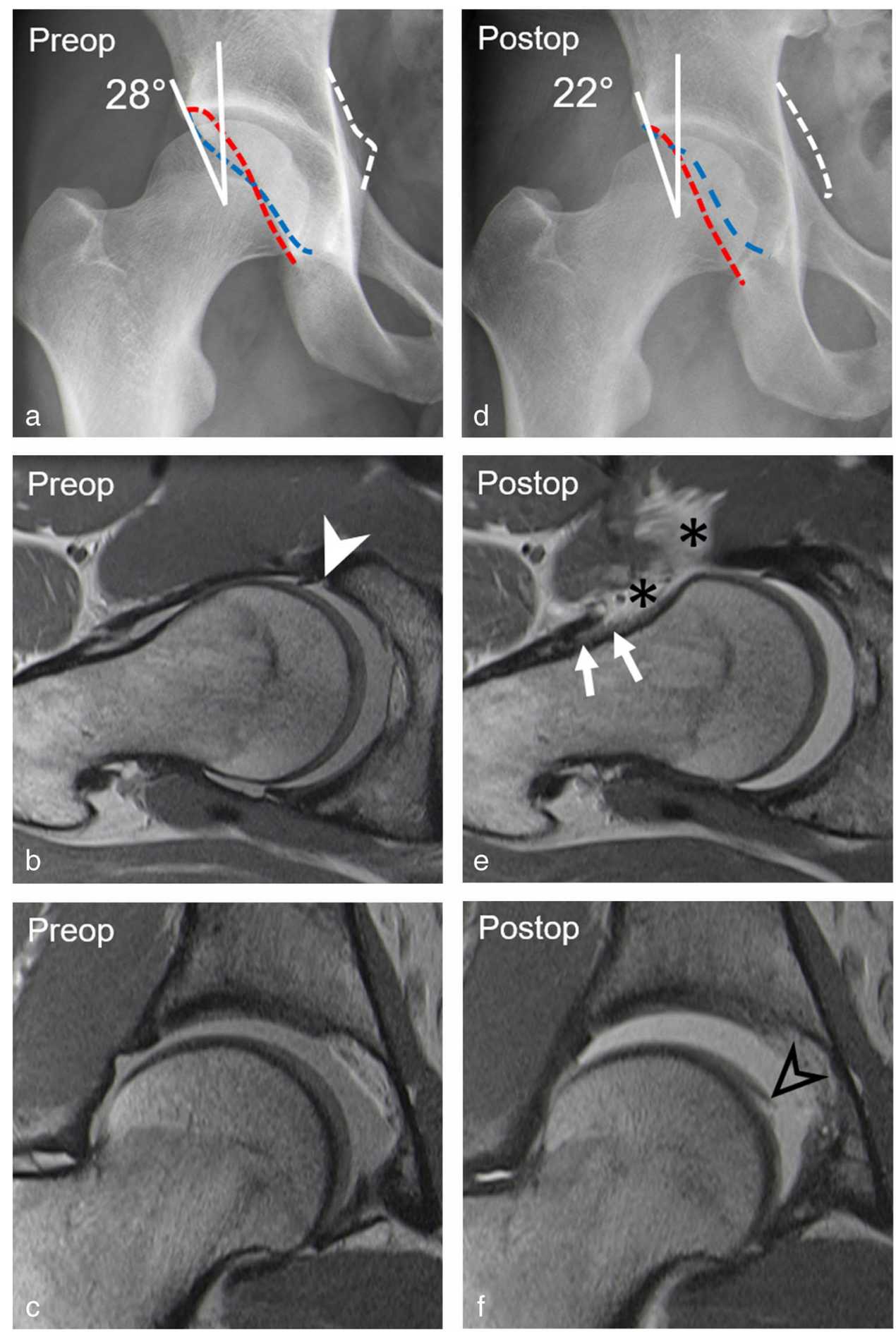

\section{Discussion}

Despite the fact that the number of patients with hip pain following arthroscopic FAI surgery are increasing secondary to the rise of hip arthroscopy, studies investigating osseous deformities and intra-articular lesions on postoperative imaging are sparse [10].
Most patients had osseous deformities of the acetabulum or femur on postoperative imaging (74\% for reader 1 and $65 \%$ for reader 2). Prevalence of hip dysplasia increased from $2 \%$ and $4 \%$ preopervatively to $20 \%$ and $22 \%$ postoperatively for two readers $(p=0.01)$, secondary to overcorrection at the acetabular rim. Cadaveric studies have shown that excessive acetabular rim trimming can dramatically increase contact pressures with potentially detremential effects even 
Fig. 3 A-26 year-old man presenting with groin pain 3 years after arthroscopic acetabular rim trimming, labrum refixation, and cam resection. a Preoperative AP pelvis view shows acetabular retroversion with normal lateral coverage (LCE: $31^{\circ}$ ) and a cam deformity. b Preoperative radial reformatted image (3D T2-w true FISP, repetition time/echo time, $4.7 \mathrm{~ms} / 2 \mathrm{~ms}$ ) shows a cam deformity (alpha angle: $\left.78^{\circ}\right)$. c Preoperative coronal MR arthrogram (T1-w TSE sequence; $450 \mathrm{~ms} / 12 \mathrm{~ms}$ ) shows no chondral damage. $\mathbf{d}$ Postoperative radiograph shows less pronounced crossover sign. e Postoperative radial image (3D PD-w SPACE, $1100 \mathrm{~ms} / 41 \mathrm{~ms}$ ) shows incomplete cam resection with residual cam deformity postero-superiorly. f Postoperative coronal T1-w TSE image (450 ms/12 ms) shows progressive cartilage damage with new acetabular (white arrowhead) and femoral (black arrowhead) chondral damage. Note: Anterior acetabular wall (red dotted line), posterior acetabular wall (blue dotted line), ischial spine sign (white dotted line), femoral head (white dotted circle)
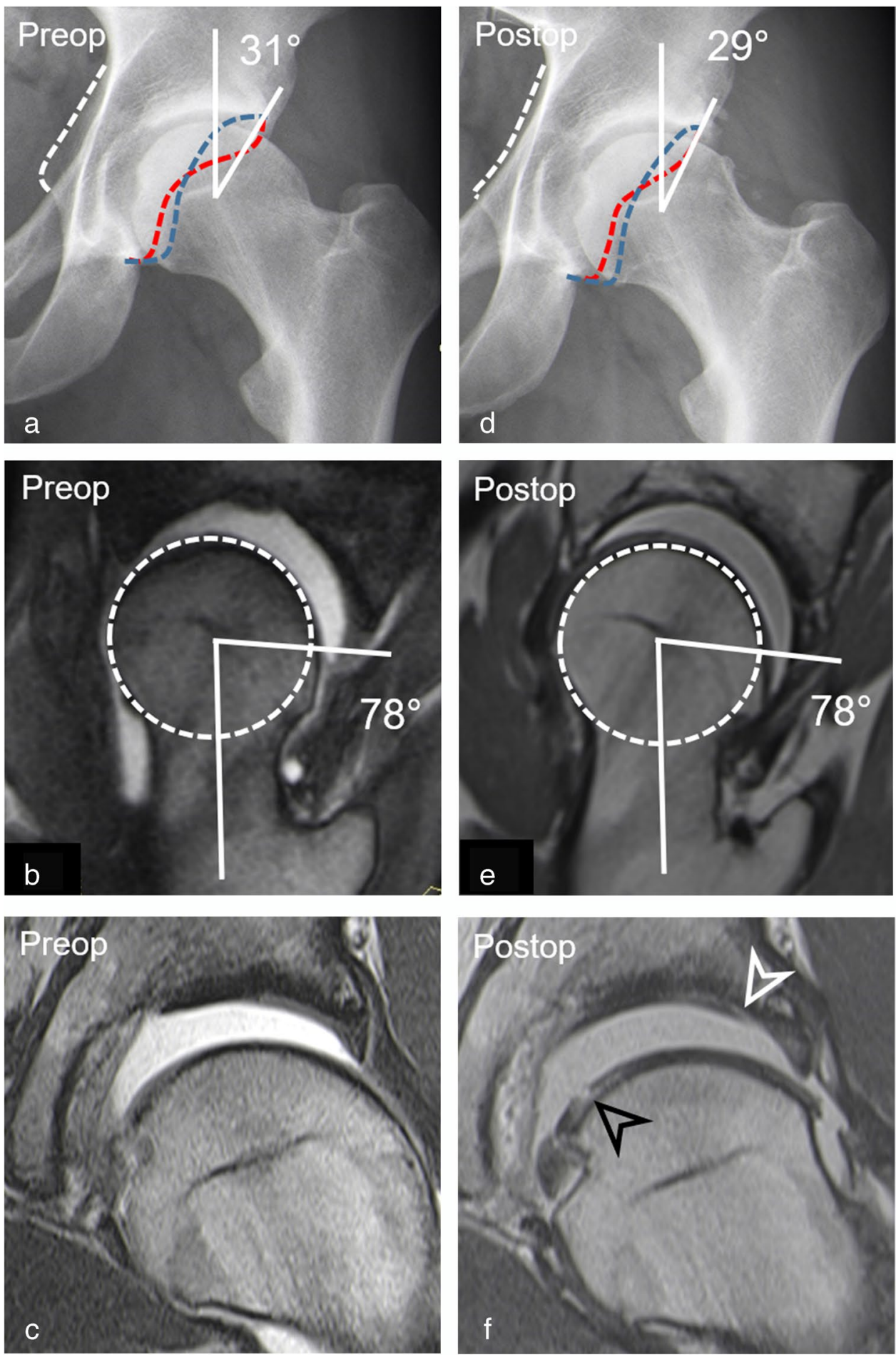

in non-dysplastic hip joints [27]. Insufficient acetabular coverage on radiographs ( $\mathrm{LCE}<22^{\circ}$, hazard ratio 5.4 ) or excessive osseous debridement on MRI reportedly increases the long-term failure rate and impairs short-term improvement after FAI surgery [2]. Identification of these patients is important as they may require subsequent periacetabular osteotomy to correct iatrogenic acetabular undercoverage [7]. In the present study, a postoperative LCE angle $<25^{\circ}$ was associated (OR 5.13, $p=0.04$ ) with the presence of postoperative labrum lesions which supposedly result from the increased stress load. In addition, excessively high femoral torsion can aggravate the overload to the acetabulum 

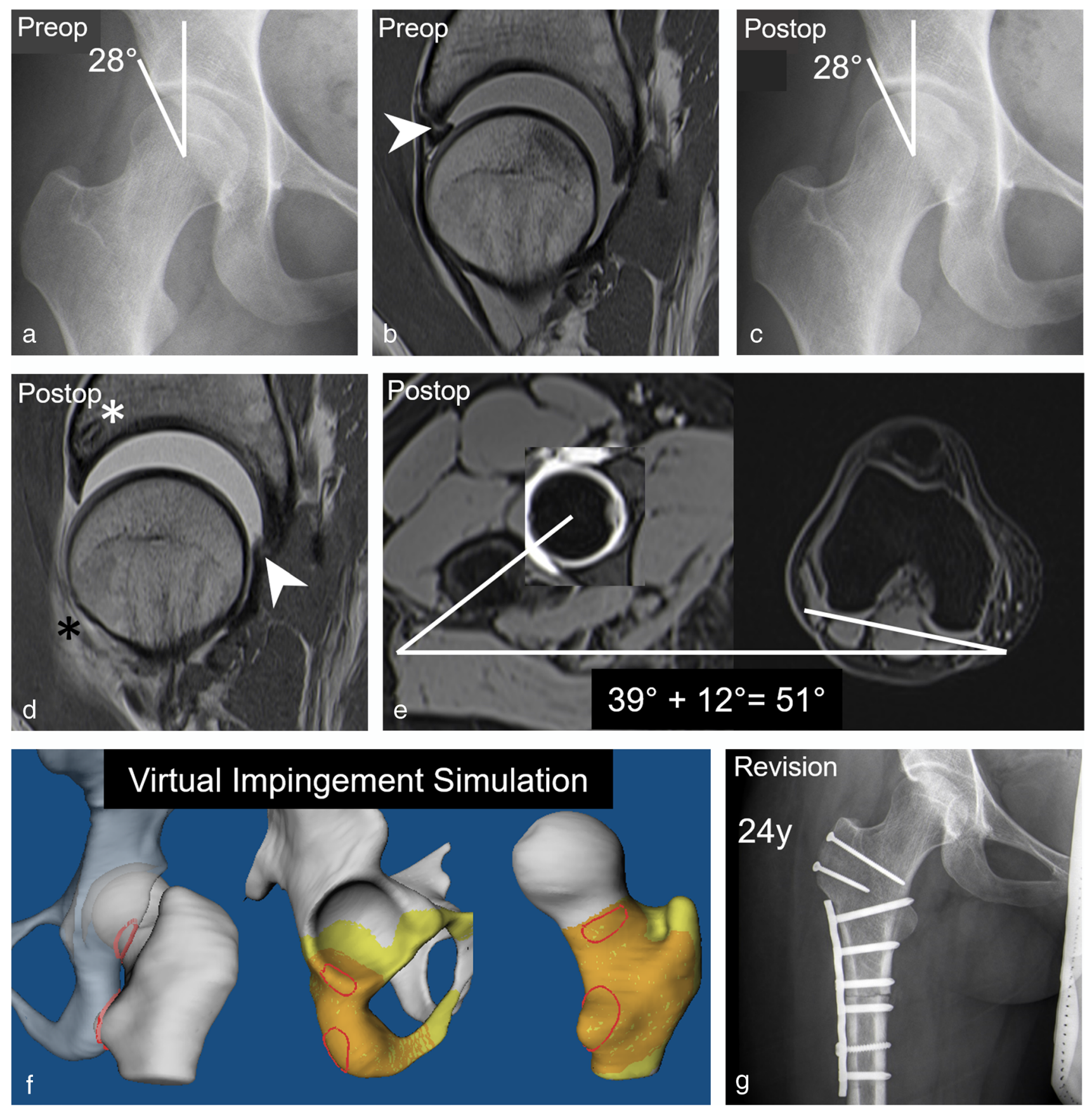

Fig. 4 A 24-year-old patient presenting with persisting pain 1.5 years after arthroscopic labrum refixation and cam resection. a Preoperative AP pelvis view shows no acetabular deformity. b Preoperative sagittal PD-w TSE image (repetition time/echo time, $2460 \mathrm{~ms} / 13 \mathrm{~ms}$ ) shows anterior labrum tear (arrowhead). c Postoperative AP pelvis view shows no obvious deformity. d Postoperative sagittal PD-w TSE image $(2460 \mathrm{~ms} / 13 \mathrm{~ms})$ shows intact anterior labrum after refixation with suture anchors (white asterisk) but new labrum tear posterior (arrowhead). Anterior capsule defect with adhesion (black aster-

due to anterior translation of the femoral head secondary to an ischiofemoral impingement conflict [28-30]. In the present study, the prevalence of exccessivley high femoral isk). e Postoperative axial T1-w VIBE dixon sequence $(6.7 \mathrm{~ms}, 2.4$ and $4.8 \mathrm{~ms}$ ) shows increased femoral torsion $\left(51^{\circ}\right)$. f Postoperative MRI-based 3D impingement simulation was performed which confirmed an ischiofemoral impingement conflict occurring at the lesser trochanter, the posterior acetabulum, and the ischial tuberosity (red areas) during combined extension, external rotation, and adduction. $\mathbf{g}$ Postoperative radiograph following subsequent open surgical hip dislocation and subtrochanteric derotational osteotomy for treatment of ischiofemoral impingement

torsion $\left(>35^{\circ}\right)$ measured at the level of the lesser trochanter was $24 \%$ for reader 1 and $20 \%$ for reader 2, considerably higher than the $12 \%$ reported in 538 patients with FAI and 
Table 3 Measure of interobserver agreement

\begin{tabular}{lllll}
\hline Parameter & $\begin{array}{l}\text { Baseline } \\
\text { K value }\end{array}$ & $p$ value & $\begin{array}{l}\text { Postoperative } \\
\text { K value }\end{array}$ & $p$ value \\
\hline Osseous deformity & & & & \\
Hip dysplasia $\left(\mathrm{LCE}<25^{\circ}\right)$ & 0.66 & $<0.001$ & 0.80 & $<0.001$ \\
Severe overcoverage $\left(\mathrm{LCE}>40^{\circ}\right)$ & 0.80 & $<0.001$ & 0.62 & $<0.001$ \\
Acetabular retroversion & 0.91 & $<0.001$ & 0.66 & $<0.001$ \\
Cam deformity & 0.67 & $<0.001$ & 0.60 & $<0.001$ \\
Increased femoral torsion $\left(>35^{\circ}\right)$ & - & - & 0.62 & $<0.001$ \\
Decreased femoral torsion $\left(<5^{\circ}\right)$ & - & - & 1.00 & $<0.001$ \\
Intra-articular lesion & & - & & $<0.001$ \\
Postoperative labrum tear & - & $<0.001$ & 0.52 & $<0.001$ \\
Acetabular cartilage lesion & 0.51 & $<0.001$ & 0.78 & $<0.001$ \\
Femoral cartilage lesion & 0.85 & - & 0.72 & $<0.001$ \\
Progressive cartilage damage & - & - & 0.70 & $<0.001$ \\
Capsular defect & - & - & 0.82 & $<0.001$ \\
Capsular adhesion & - & &
\end{tabular}

$L C E$ lateral center edge angle

Table 4 Association between demographic factors and imaging parameters with progressive cartilage damage on postoperative MR arthrograms

\begin{tabular}{|c|c|c|c|c|c|c|c|}
\hline \multirow[t]{2}{*}{ Parameter } & \multicolumn{7}{|c|}{ Progressive cartilage damage } \\
\hline & $\begin{array}{l}\text { Yes } \\
(n=17)\end{array}$ & $\begin{array}{l}\text { No } \\
(n=29)\end{array}$ & Difference & Odds ratio univariate & $p$ value & Odds ratio multivariate & $p$ value \\
\hline Age & $31 \pm 10$ & $28 \pm 9$ & $3(-3-9)$ & $1.04(0.97-1.11)$ & 0.256 & & \\
\hline Preoperative cartilage lesion $>2 \mathrm{~h}$ & $41 \%(7)$ & $14 \%(4)$ & $27 \%(14-40 \%)$ & $4.38(1.08-20.02)$ & 0.038 & $7.72(1.52-50.28)$ & 0.02 \\
\hline PreoperativeTönnis > 0 & $47 \%(8)$ & $21 \%(6)$ & $26 \%(13-39 \%)$ & $3.41(0.94-13.24)$ & 0.067 & & \\
\hline Postoperative LCE $\left({ }^{\circ}\right)$ & $29 \pm 7$ & $32 \pm 6$ & $3(-1-7)$ & $0.97(0.87-1.07)$ & 0.536 & & \\
\hline Dysplasia $\left(\mathrm{LCE}<25^{\circ}\right)$ & $35 \%(6)$ & $10 \%(3)$ & $25 \%(13-38 \%)$ & $4.73(1.05-25.77)$ & 0.043 & $6.14(0.89-58.64)$ & 0.08 \\
\hline Severe overcoverage $\left(\mathrm{LCE}>40^{\circ}\right)$ & $12 \%(2)$ & $14 \%(4)$ & $2 \%(-2-6 \%)$ & $0.83(0.11-4.82)$ & 0.844 & & \\
\hline Postoperative acetabular retroversion & $6 \%(1)$ & $0 \%(0)$ & $6 \%(-1-13)$ & n.a & n.a & & \\
\hline Postoperative cam deformity & $47 \%(8)$ & $17 \%(5)$ & $30 \%(17-43 \%)$ & $4.27(1.13-17.67)$ & 0.032 & $0.09(0.00-3.53)$ & 0.20 \\
\hline Alpha angle $\left({ }^{\circ}\right)$ & $66 \pm 11$ & $57 \pm 10$ & $9(3-16)$ & $1.09(1.02-1.17)$ & 0.005 & $1.18(1.03-1.44)$ & 0.04 \\
\hline Postoperative femoral torsion $\left({ }^{\circ}\right)$ & $22 \pm 11$ & $25 \pm 10$ & $3(-4-9)$ & $0.98(0.92-1.03)$ & 0.402 & & \\
\hline Increased femoral torsion $\left(>35^{\circ}\right)$ & $18 \%(3)$ & $28 \%(8)$ & $10 \%(1-19 \%)$ & $0.56(0.11-2.33)$ & 0.438 & & \\
\hline Decreased femoral torsion $\left(<0^{\circ}\right)$ & $12 \%(2)$ & $0 \%(0)$ & $12 \%(3-21 \%)$ & n.a & n.a & & \\
\hline Postoperative capsular defect & $29 \%(5)$ & $59 \%(17)$ & $30 \%(17-43 \%)$ & $0.29(0.08-1.02)$ & 0.052 & & \\
\hline Postoperative adhesions & $41 \%(7)$ & $38 \%(11)$ & $3 \%(-2-8 \%)$ & $1.15(0.33-3.90)$ & 0.828 & & \\
\hline
\end{tabular}

$L C E$ lateral center edge angle

hip dysplasia using the same measurement method [31]. Although indications are still evolving, early studies report favorable outcome following derotational osteotomies for treatment of ischiofemoral impingement and instability [29]. Defects of the hip capsule represent another potentially destabilizing factor secondary to hip arthroscopy. Identification of capsular defects is important as some patients may require revision surgery for capsular repair [32]. Similar to previous reports, the prevalence of capsular defects was high with $48 \%$ and $59 \%$ for two readers [11, 32].

Restoring a spherical femoral head neck junction is critical to achieve maximum clinical short-term outcome and residual cam deformities reportedly are among the most frequent causes for revision surgery [12, 33]. Alpha angles decreased from $71 \pm 9^{\circ}$ and $70 \pm 11^{\circ}$ preoperatively to $60 \pm 11^{\circ}$ and $60 \pm 10^{\circ}$ postoperatively for readers 1 and 
Fig. 5 A 45-year-old patient presenting with aggravated groin pain 1 year after arthroscopic cam resection and labrum debridement. a Preoperative AP pelvis view shows normal lateral coverage (LCE: $26^{\circ}$ ) and a cam deformity but a preserved joint space. b Preoperative radial reformatted image (3D T2-w true FISP, repetition time/echo time, $4.7 \mathrm{~ms} / 2 \mathrm{~ms}$ ) shows superior cam deformity (alpha angle: $82^{\circ}$ ). c Preoperative coronal T1-w TSE image (450 ms/12 ms) shows extensive cartilage damage of the central acetabulum (white arrowhead) and labrum tear (filled arrowhead). d Postoperative AP pelvis view with normal acetabular coverage (LCE: $26^{\circ}$ ) and joint space narrowing. $\mathbf{e}$ Postoperative radial reformatted image (3D T2-w true FISP, $4.7 \mathrm{~ms} / 2 \mathrm{~ms}$ ) shows residual cam deformity due to undercorrection (alpha angle: $82^{\circ}$ ). f Postoperative T1-w TSE image (450 ms/ and $12 \mathrm{~ms}$ ) shows complete loss of acetabular cartilage (white arrowheads) and new femoral cartilage thinning (black arrowhead) indicating progressive cartilage damage. Residual labrum tear (filled arrowhead). g Postoperative AP pelvis view following total hip replacement 8 months after postoperative MR arthrogram. Note: femoral head (white dotted circle)
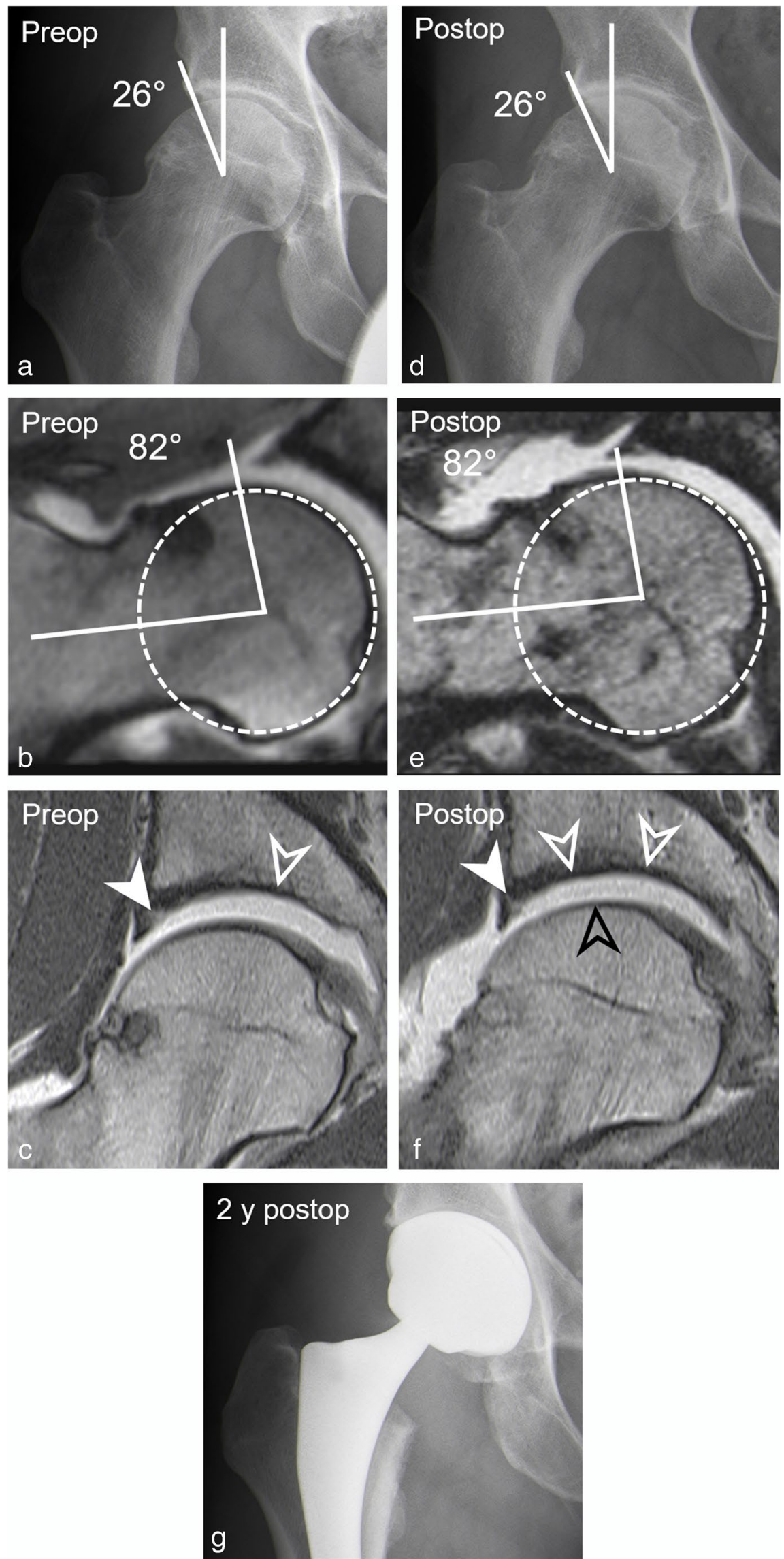
Table 5 Association between demographic factors and imaging parameters with postoperative labrum lesions on postoperative MR arthrograms

\begin{tabular}{|c|c|c|c|c|c|}
\hline \multirow[t]{2}{*}{ Parameter } & \multicolumn{5}{|c|}{ Postoperative labrum lesion } \\
\hline & Yes $(n=22)$ & No $(n=24)$ & Difference & Odds ratio univariate & $p$ value \\
\hline Age & $31 \pm 10$ & $27 \pm 10$ & $4(-3-9)$ & $1.034(0.97-1.10)$ & 0.28 \\
\hline Preoperative cartilage lesion $>2 \mathrm{~h}$ & $36 \%(8)$ & $13 \%(3)$ & $23 \%(11-35)$ & $4.00(0.97-20.76)$ & 0.06 \\
\hline Preoperative Tönnis $>0$ & $41 \%(9)$ & $21 \%(5)$ & $20 \%(8-32 \%)$ & $2.63(-0.31-2.33)$ & 0.14 \\
\hline Postoperative LCE $\left(^{\circ}\right)$ & $30 \pm 8$ & $31 \pm 5$ & $1(-5-3)$ & $0.99(0.89-1.07)$ & 0.62 \\
\hline Dysplasia $\left(\mathrm{LCE}<25^{\circ}\right)$ & $32 \%(7)$ & $8 \%(2)$ & $24 \%(12-36 \%)$ & $5.13(1.07-37.76)$ & 0.04 \\
\hline Severe overcoverage $\left(\mathrm{LCE}>40^{\circ}\right)$ & $14 \%(3)$ & $13 \%(3)$ & $1 \%(-2-4 \%)$ & $1.11(0.18-6.61)$ & 0.91 \\
\hline Postoperative acetabular retroversion & $5 \%(1)$ & $0 \%(0)$ & $5 \%(-1-11 \%)$ & n.a & n.a \\
\hline Postoperative cam deformity & $32 \%(7)$ & $25 \%(6)$ & $7 \%(0-14 \%)$ & $1.40(-0.96-1.66)$ & 0.61 \\
\hline Alpha angle $\left({ }^{\circ}\right)$ & $61 \pm 11$ & $60 \pm 11$ & $1(-7-6)$ & $1.01(0.95-1.06)$ & 0.82 \\
\hline Postoperative femoral torsion $\left({ }^{\circ}\right)$ & $24 \pm 11$ & $24 \pm 10$ & 0 & $1.00(0.95-1.06)$ & 0.99 \\
\hline Increased femoral torsion $\left(>35^{\circ}\right)$ & $18 \%(4)$ & $29 \%(7)$ & $11 \%(2-20 \%)$ & $0.54(0.12-2.12)$ & 0.38 \\
\hline Decreased femoral torsion $\left(<0^{\circ}\right)$ & $5 \%(1)$ & $4 \%(1)$ & $1 \%(-2-4 \%)$ & n.a & n.a \\
\hline Postoperative capsular defect & $41 \%(9)$ & $54 \%(13)$ & $13 \%(3-23 \%)$ & $0.59(0.18-1.87)$ & 0.37 \\
\hline Postoperative adhesions & $41 \%(9)$ & $38 \%(9)$ & $3 \%(-2-8 \%)$ & $1.15(0.353 .82)$ & 0.81 \\
\hline
\end{tabular}

LCE lateral center edge angle

$2(p<0.001)$ following cam resection. Accordingly, 28\% (reader 1) and 22\% (reader 2) of the patients had a residual cam deformity using the commonly recommended threshold of $60^{\circ}$ for the alpha angle [20]. The present study underlines the importance of a complete cam resection as an increased odds for progression of cartilage damage was observed with increasing alpha angles (OR 1.18, $p=0.04$ ). Previously Ross et al. reported a mean alpha angle of $68 \pm 16^{\circ}$ corresponding to a $86 \%$ prevalence of residual cam deformity when applying a threshold of $50^{\circ}$ in 50 patients undergoing revision hip arthroscopy. The authors stressed the importance of using dynamic virtual impingement simulation in these patients to take the entire hip anatomy into account [12]. This further includes measurement of femoral torsion as reduced femoral torsion can cause hip impingement in the absence of a cam deformity and has been reported as a risk factor for worse clinical outcome following arthroscopic FAI surgery [34, 35].

Manifest radiographic osteoarthritis is an established negative predictor for success of joint preserving surgery but pre-arthritic to early degenerative radiographic changes fail in predicting severity of already present cartilage damage [20]. Previously, it has been shown that extensive cartilage damage $>2 \mathrm{~h}$ on the clock face is associated with an increase risk (hazard ratio 4.6) of failure following open FAI surgery [24]. In the present study, extensive cartilage damage $>2 \mathrm{~h}$ was associated with increased odds (OR 7.72, $p=0.02$ ) for progressive cartilage damage on postoperative MR arthrograms. This underlines the potential benefit of a detailed reporting of cartilage damage in risk stratification and surgical decision-making. In the current study, inter-reader reliabilites for imaging assessessment ranged from moderate to almost perfect ( $\kappa=0.51$ to 1$)$ which is similar to a previous study on postoperative MR arthrographic findings after FAI surgery which reported fair to almost perfect agreement between readers ( $\kappa=0.25$ to 1$)$ [11].

Our study has several limitations. First, due to ethical reasons, we did not include an asymptomatic control group following hip arthroscopy who underwent MR arthrography of the hip. Previous studies have shown that chondro-labral damage and capsular lesions are highly prevalent on postoperative MRI in patients with good clinical outcome [10,11]. More specifically, Kim et al. demonstrated that the prevalence of intra-articular lesions on postoperative MR arthrography including chondro-labral and capsular lesions was comparable between patients with and without pain 13 months after arthroscopic FAI correction [11]. However, comparing pre- and postoperative MRI after arthroscopic FAI correction, Foreman et al. could show that increased depth and length of the acetabuloplasty was associated with decreased short-term improvement in pain scores.[10]. Second, due to the retrospective study design, patients did not complete pre- and postoperative questionnaires. Hence, it was not possible to assess changes in patientreported outcome scores to evaluate which imaging findings are associated with worse clinical outcome. Overall, this highlights the importance of correlating postoperative imaging findings with clinical presentation in a given patient and the need for further research investigating their association. Third, assessment of intraarticular lesions was based on traction MR arthrography. 

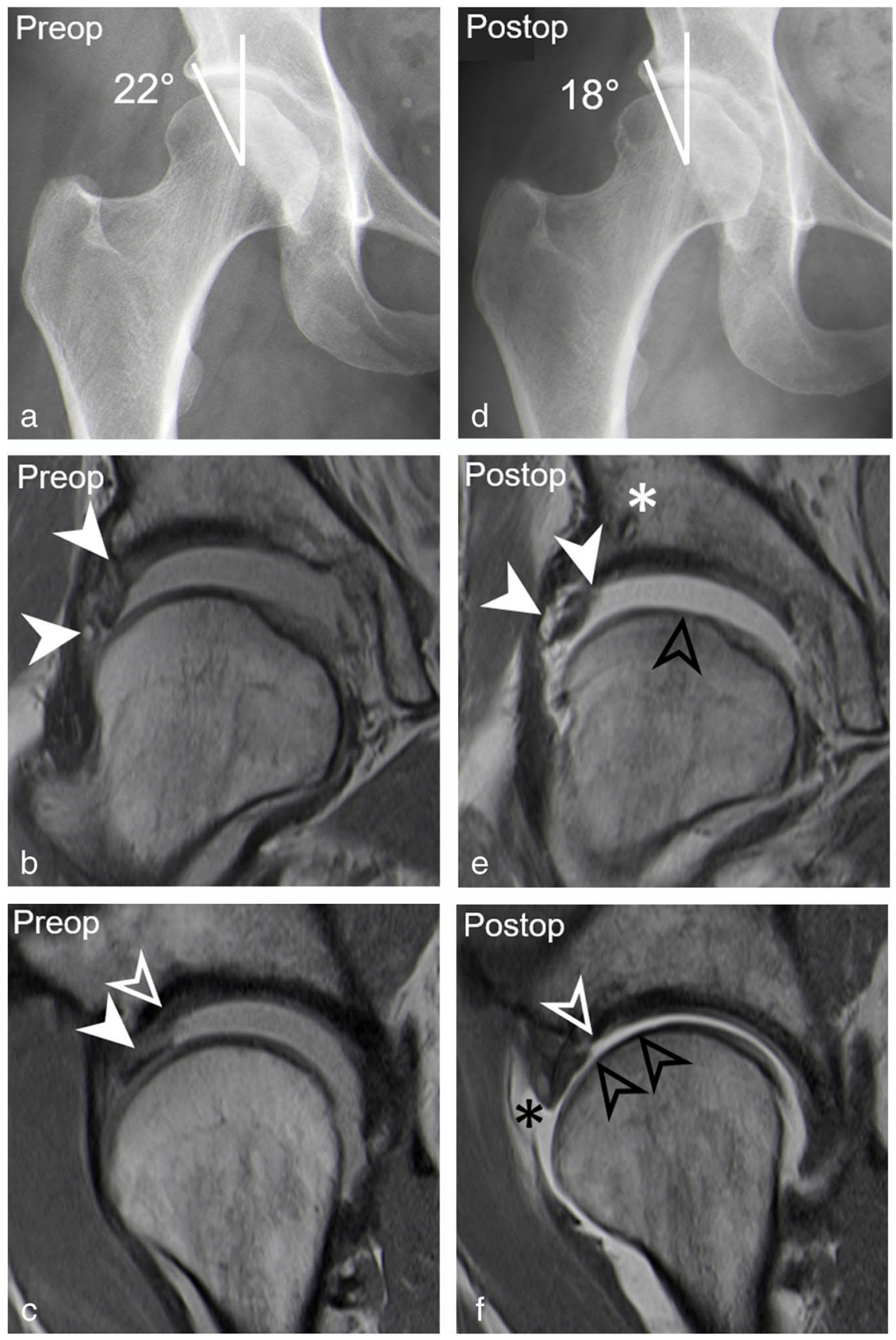

Fig. 6 A 38-year-old woman presenting with persisting pain 1 year after arthroscopic acetabular rim trimming and labrum refixation. a Preoperative AP pelvis view shows dysplasia with decreased lateral coverage (LCE: $22^{\circ}$ ). b Preoperative coronal PD-w TSE image (repetition time/echo time, 2460/13 ms) shows labrum tear with paralabral cyst (filled arrowheads). c Preoperative sagittal PD-w TSE image $(2460 \mathrm{~ms} / 13 \mathrm{~ms})$ shows extensive acetabular cartilage delamination (white arrowhead) with adjacent labrum tear (filled arrowhead). d Postoperative AP pelvis view shows further reduced lateral coverage (LCE: $18^{\circ}$ ) after acetabular rim trimming. e Postoperative coronal PD-w TSE image (2460/13 ms) shows retear of labrum (filled arrowheads) following labrum refixation with suture anchors (asterisk) and new femoral cartilage thinning (black arrowhead). f Postoperative

sagittal PD-w TSE image (2460 ms/13 ms) shows excessive acetabular cartilage defect (white arrowhead), new femoral cartilage defect (black arrowheads), and a capsular defect (black asterisk). g Postoperative axial T1-w TSE image $(540 \mathrm{~ms} / 19 \mathrm{~ms})$ and axial T1-w VIBE DIXON of the knee $(6.7 \mathrm{~ms} / 2.4$ and $4.8 \mathrm{~ms})$ show excessively high femoral torsion of $44^{\circ}$. $\mathbf{h}$ At revision hip arthroscopy the retear of the labrum (L) was confirmed with loose sutures (white arrowheads) and acetabular (AC) and femoral (FC) chondral damage. i Intraoperative remnants of the anterior joint capsule $(\mathrm{C})$ can be seen along with an intraarticular course of the psoas tendon (PT). $\mathbf{j}$ The patient had persisting pain following revision hip arthroscopy and underwent subsequent total hip replacement 

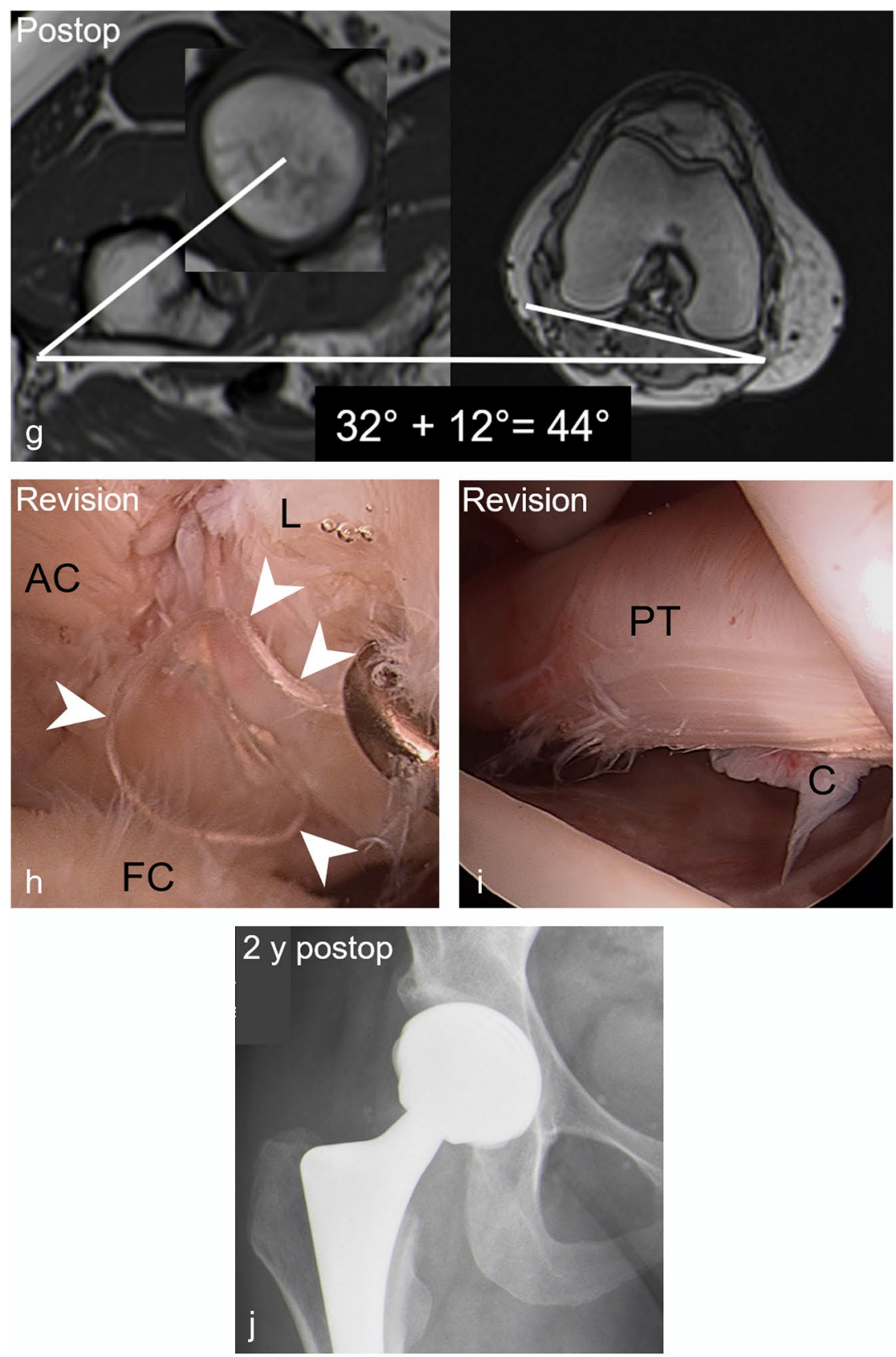

Fig. 6 (continued)

While leg traction reportedly improves visualization of cartilage layers, the subluxation of the femoral head can make identification of capsular lesions more difficult [18]. Ideally, a direct comparison between images obtained with and without traction should be performed. which was not possible in a busy clinical setup and should be assessed in future studies.

To conclude, we found a high prevalence of osseous deformities due to over- or undercorrrection of the hip and a high prevalence of associated intra-articular lesions in patients following failed hip arthroscopy. Detection of these deformities is important as they may expose the hip to ongoing stress and can potentially lead to progressive cartilage damage and labrum lesions.

Supplementary Information The online version contains supplementary material available at https://doi.org/10.1007/s00330-021-08398-4. 
Funding Open access funding provided by University of Bern. The authors state that this work has not received any funding.

\section{Declarations}

Guarantor The scientific guarantor of this publication is Florian Schmaranzer, MD PhD.

Conflict of interest The authors of this manuscript declare no relationships with any companies, whose products or services may be related to the subject matter of the article.

Statistics and biometry One of the authors has significant statistical expertise.

Informed consent Written informed consent was waived by the Institutional Review Board.

Ethical approval Institutional Review Board approval was obtained.

\section{Methodology • retrospective}

- observational study

- performed at one institution

Open Access This article is licensed under a Creative Commons Attribution 4.0 International License, which permits use, sharing, adaptation, distribution and reproduction in any medium or format, as long as you give appropriate credit to the original author(s) and the source, provide a link to the Creative Commons licence, and indicate if changes were made. The images or other third party material in this article are included in the article's Creative Commons licence, unless indicated otherwise in a credit line to the material. If material is not included in the article's Creative Commons licence and your intended use is not permitted by statutory regulation or exceeds the permitted use, you will need to obtain permission directly from the copyright holder. To view a copy of this licence, visit http://creativecommons.org/licenses/by/4.0/.

\section{References}

1. Sing DC, Feeley BT, Tay B, Vail TP, Zhang AL (2015) Agerelated trends in hip arthroscopy: a large cross-sectional analysis. Arthroscopy 31(12):2307-2313.e2

2. Steppacher SD, Anwander H, Zurmühle CA, Tannast M, Siebenrock KA (2015) Eighty percent of patients with surgical hip dislocation for femoroacetabular impingement have a good clinical result without osteoarthritis progression at 10 years. Clin Orthop Relat Res. 473(4):1333-1341

3. Griffin DR, Dickenson EJ, Wall PDH et al (2018) Hip arthroscopy versus best conservative care for the treatment of femoroacetabular impingement syndrome (UK FASHIoN): a multicentre randomised controlled trial. Lancet 391(10136):2225-2235

4. Schmaranzer F, Kheterpal AB, Bredella MA (2021) Best practices: hip femoroacetabular impingement. AJR Am J Roentgenol 216(3):585-598

5. Baron JE, Westermann RW, Bedard NA, Willey MC, Lynch TS, Duchman KR (2020) Is the actual failure rate of hip arthroscopy higher than most published series? An Analysis of a Private Insurance Database. Iowa Orthop J 40(1):135-142

6. Mygind-Klavsen B, Nielsen TG, Lund B, Lind M (2021) Clinical outcomes after revision hip arthroscopy in patients with femoroacetabular impingement syndrome (FAIS) are inferior compared to primary procedures. Results from the Danish Hip
Arthroscopy Registry (DHAR). Knee Surg Sports Traumatol Arthrosc 29(4):1340-1348

7. Shapira J, Kyin C, Go C et al (2020) Indications and outcomes of secondary hip procedures after failed hip arthroscopy: a systematic review. Arthroscopy 36(7):1992-2007

8. Dietrich TJ, Dora C, Pfirrmann CWA (2013) Postoperative imaging in femoroacetabular impingement. Semin Musculoskelet Radiol 17(3):272-278

9. Hanke MS, Lerch TD, Schmaranzer F, Meier MK, Steppacher SD, Siebenrock KA (2021) Complications of hip preserving surgery. EFORT Open Rev 6(6):472-486

10. Foreman SC, Zhang AL, Neumann J et al (2020) Postoperative MRI findings and associated pain changes after arthroscopic surgery for femoroacetabular impingement. AJR Am J Roentgenol 214(1):177-184

11. Kim CO, Dietrich TJ, Zingg PO, Dora C, Pfirrmann CWA, Sutter R (2017) Arthroscopic hip surgery: frequency of postoperative MR arthrographic findings in asymptomatic and symptomatic patients. Radiology 283(3):779-788

12. Ross JR, Larson CM, Adeoye O, Kelly BT, Bedi A (2015) Residual deformity is the most common reason for revision hip arthroscopy: a three-dimensional CT study. Clin Orthop Relat Res 473(4):1388-1395

13. McCarthy JC, Glassner PJ (2013) Correlation of magnetic resonance arthrography with revision hip arthroscopy. Clin Orthop Relat Res 471(12):4006-4011

14. Haefeli PC, Schmaranzer F, Steppacher SD, Cullmann JL, Tannast M, Büchler L (2018) Imaging appearance and distribution of intra-articular adhesions following open FAI surgery. Eur J Radiol 104:71-78

15. Blankenbaker DG, De Smet AA, Keene JS (2011) MR arthrographic appearance of the postoperative acetabular labrum in patients with suspected recurrent labral tears. AJR Am J Roentgenol 197(6):W1118-1122

16. Tannast M, Siebenrock KA, Anderson SE (2007) Femoroacetabular impingement: radiographic diagnosis-what the radiologist should know. AJR Am J Roentgenol 188(6):1540-1552

17. Schmaranzer F, Klauser A, Kogler M et al (2015) Diagnostic performance of direct traction MR arthrography of the hip: detection of chondral and labral lesions with arthroscopic comparison. Eur Radiol 25(6):1721-1730

18. Schmaranzer F, Klauser A, Kogler M et al (2014) Improving visualization of the central compartment of the hip with direct MR arthrography under axial leg traction: a feasibility study. Acad Radiol 21(10):1240-1247

19. Wiberg G (1939) Studies on dysplastic acetabula and congenital subluxation of the hip joint : with special reference to the complication of osteoarthritis. Acta Chir Scand 83(Suppl 58):53-68

20. Mascarenhas VV, Castro MO, Rego PA et al (2020) The Lisbon Agreement on Femoroacetabular Impingement Imaging-part 1: overview. Eur Radiol 30(10):5281-5297

21. Murphy SB, Simon SR, Kijewski PK, Wilkinson RH, Griscom NT (1987) Femoral anteversion. J Bone Joint Surg Am 69(8):1169-1176

22. Schmaranzer F, Kallini JR, Miller PE, Kim Y-J, Bixby SD, Novais EN (2020) The effect of modality and landmark selection on MRI and CT femoral torsion angles. Radiology 296(2):381-390

23. Schmaranzer F, Todorski IAS, Lerch TD, Schwab J, CullmannBastian J, Tannast M (2017) Intra-articular lesions: imaging and surgical correlation. Semin Musculoskelet Radiol 21(5):487-506

24. Hanke MS, Steppacher SD, Anwander H, Werlen S, Siebenrock KA, Tannast M (2017) What MRI findings predict failure 10 years after surgery for femoroacetabular impingement? Clin Orthop Relat Res 475(4):1192-1207

25. Landis JR, Koch GG (1977) The measurement of observer agreement for categorical data. Biometrics 33(1):159-174 
26. Donner A, Eliasziw M (1992) A goodness-of-fit approach to inference procedures for the kappa statistic: confidence interval construction, significance-testing and sample size estimation. Stat Med 11(11):1511-1519

27. Bhatia S, Lee $S$, Shewman E et al (2015) Effects of acetabular rim trimming on hip joint contact pressures: how much is too much? Am J Sports Med 43(9):2138-2145

28. Chaharbakhshi EO, Hartigan DE, Perets I, Domb BG (2019) Is hip arthroscopy effective in patients with combined excessive femoral anteversion and borderline dysplasia? A Match-Controlled Study. Am J Sports Med 47(1):123-130

29. Lerch TD, Schmaranzer F, Steppacher SD, Ziebarth K, Tannast M, Siebenrock KA (2020) Most of patients with femoral derotation osteotomy for posterior extraarticular hip impingement and high femoral version would do surgery again. Hip Int. https://doi.org/ $10.1177 / 1120700020953100$.

30. Lerch TD, Zwingelstein S, Schmaranzer F et al (2021) Posterior extra-articular ischiofemoral impingement can be caused by the lesser and greater trochanter in patients with increased femoral version: dynamic 3D CT-based hip impingement simulation of a modified FABER test. Orthop J Sports Med 9(5):2325967121990629

31. Lerch TD, Todorski IAS, Steppacher SD et al (2018) Prevalence of femoral and acetabular version abnormalities in patients with symptomatic hip disease: a controlled study of 538 hips. Am J Sports Med 46(1):122-134
32. Owens JS, Jimenez AE, Shapira J et al (2021) Capsular repair may improve outcomes in patients undergoing hip arthroscopy for femoroacetabular impingement: a systematic review of comparative outcome studies. Arthroscopy 37(9):2975-2990

33. Lansdown DA, Kunze K, Ukwuani G, Waterman BR, Nho SJ (2018) The importance of comprehensive cam correction: radiographic parameters are predictive of patient-reported outcome measures at 2 years after hip arthroscopy. Am J Sports Med 46(9):2072-2078

34. Fabricant PD, Fields KG, Taylor SA, Magennis E, Bedi A, Kelly BT (2015) The effect of femoral and acetabular version on clinical outcomes after arthroscopic femoroacetabular impingement surgery. J Bone Joint Surg Am 97(7):537-543

35. Lerch TD, Boschung A, Todorski IAS et al (2019) Femoroacetabular impingement patients with decreased femoral version have different impingement locations and intra- and extraarticular anterior subspine FAI on 3D-CT-based impingement simulation: implications for hip arthroscopy. Am J Sports Med 47(13):3120-3132

Publisher's note Springer Nature remains neutral with regard to jurisdictional claims in published maps and institutional affiliations. 